\title{
Devenir à long terme d'exploitations abandonnées de sel
}

\section{GHOREYCHI X. DAUPLEY}

INERIS

Parc technologique Alata

$B P n^{\circ} 2$

60550 Vermeuil-en-Halatte

www.ineris.fr

Mehdi.Ghoreychi@ineris.fr

Xavier.Daupley@ineris.fr
'으 L'évolution à long terme des sites d'exploitation

abandonnée de sel est discutée en analysant trois aspects essentiels: les contextes géologique et hydrogéologique du gisement de sel, les caractéristiques de l'exploitation (méthodes, géométries et dimensions) et le comportement spécifique du sel gemme sous l'effet des phénomènes tels que le fluage, l'endommagement, la perméabilité et l'interaction avec les fluides notamment la saumure. Cette discussion est placée en particulier dans le contexte des exploitations de sel de Lorraine (France), réalisées par la méthode des chambres et piliers abandonnés ou par dissolution.

Mots-clés: mine, sel, stabilité mécanique, long terme, dissolution, saumure.

\section{Long term evolution of abandoned exploitations of salt}

Long term evolution of abandoned exploitations of salt is discussed by analysing three basic aspects : geological and hydro-geological contexts of salt deposit, exploitation features (methods, geometries and dimensions) and specific mechanical behaviour of salt under the effect of phenomena such as creep. damage, permeability and fluid interaction in particular brine effect. This discussion is mainly placed in the context of salt exploitations in Lorraine (France) carried out by room and pillar method or by solution mining.

Key words : mine, salt, mechanical stability, long term, solution, brine. 


\section{Introduction}

De nombreux gisements de sel exploités en France, notamment au cours des deux derniers siècles, ont déjà connu ou connaitront progressivement une cessation de leurs activités. Cette situation conduira, dans les années. à venir, à un nombre croissant d'exploitations abandonnées de sel. Dans ce contexte, on peut s'interroger sur l'évolution à long terme des sites concernés d'un point de vue de leur impact sur la sécurité des personnes et des biens et sur la protection de l'environnement. Cette interrogation est motivée par plusieurs faits

1) les cavités réalisées dans le sel ne sont pas toujours accessibles à l'homme. Cé n'est évidemment jamais le cas d'une cavité réalisée par dissolution à partir de sondages. D'autres exploitations, jadis effectuées par des techniques minières classiques et donc initialement accessibles, furent envahies accidentellement par de l'eau douce ou salée (saumure provenant de nappes salées). Dans ces conditions, des renseignements essentiels sur la forme, les dimensions, l'état de stabilité mécanique, voire même l'existence ou l'absence de cavités dans le sous-sol ne sont pas toujours disponibles. Cela s'explique d'une part par le fait que les techniques telles que le sonar, aujourd'hui largement employées avec succès pour suivre l'évolution de la forme et des dimensions des cavités de dissolution, notamment au cours de leur réalisation, n'ont été mises en œuvre que ces dernières décennies. D'autre part, les techniques de reconnaissance de vides souterrains, notamment géophysiques, qui connaissent actuellement un progrès indéniable, ne permettent pas encore de révéler, avec certitude, l'existence et l'état des cavités souterraines indépendamment de leur profondeur et de leur contexte lithologique. Dans ce cas, des renseignements obtenus à partir d'archives (plans, rapports...) deviennent précieux, bien que la quantité et la qualité de ce type de données varient largement selon le contexte et l'âge de l'exploitation:

2) le sel gemme présente un comportement mécanique spécifique, typique des évaporites : une aptitude au fluage qui se poursuit sans cesse dès lors que l'état d'équilibre naturel du matériau au repos est perturbé, par exemple, par le creusement d'un ouvrage. Ainsi, la paroi de toute cavité réalisée dans le sel est condamnée à converger plus ou moins lentement jusqu'à sa fermeture totale. Ce phénomène peut être responsable d'un affaissement différé de la surface du sol. L'amplitude et la vitesse de ce dernier dépendent de la profondeur de l'ouvrage souterrain et de la nature du recouvrement. L'affaissement résiduel susceptible de se produire à long terme (à l'échelle du siècle pour fixer les idées) est en principe lent mais il ne peut pas être évité tant qu'un espace vide souterrain est présent dans le sel. Il convient donc de s'assurer que ce mouvement différé n'est pas de nature à compromettre la stabilité à long terme des ouvrages de surface déjà existants ou réalisés après l'abandon de l'exploitation. Un facteur important est la capacité des terrains de recouvrement à encaisser les déformations imposées par le fluage du sel. Pour un recouvrement formé de terrains déformables (tels les matériaux argileux plus ou moins plastiques), le fluage de sel se traduit par un affaissement ( souple » de la surface du sol. En revanche, lorsqu'un banc raide (dolomie, anhydrite...) est présent au sein du recouvrement, ce banc peut, dans certaines conditions, se rompre sous l'effet des sollicitations imposées par le fluage du sel et entrainer l'effondrement brutal du recouvrement;

3) le sel gemme est très soluble dans l'eau (environ $350 \mathrm{~g} / \mathrm{l}$ à température ambiante et à pression atmosphérique). Sa cinétique de dissolution est également rapide. Il y a donc lieu de craindre le risque d'une dissolution à long terme de la partie non exploitée du gisement de sel si celle-ci est mise en communication avec les aquifères sus ou sous-jacents par l'intermédiaire d'une faille, d'une fissuration induite par l'exploitation ou encore d'une perméabilité naturelle de terrains. L'éventualité d'une invasion d'eau dépend en plus des caractéristiques hydrogéologiques des niveaux aquifères (caractéristiques hydrodynamiques, salinité, gradients hydrauliques des nappes...). Dans certaines conditions, la possibilité d'une dissolution progressive du sel et sa dilution dans les eaux souterraines et/ou celles de surface est réelle. Ce qui peut provoquer une pollution des réserves en eau et la création de nouveaux vides susceptibles de générer des effets en surface du sol,

On conçoit donc que l'évolution à long terme des sites affectés par l'exploitation de sel dépende de trois facteurs essentiels: les contextes géologique et hydrogéologique du gisement, les caractéristiques de l'exploitation (méthode, géométries et dimensions) et le comportement et les propriétés spécifiques du sel gemme (fluage, endommagement, perméabilité, influence de la saumure...). Ces aspects sont discutés dans la présente communication.

\section{2}

\section{Contextes géologique et hydrogéologique}

Les gisements de sel français ont fait l'objet de nombreux travaux de reconnaissance, notamment au cours des deux derniers siècles (Fries et al., 1983; Marchal, 1987; Moretto, 1987; Blanc-Valleron, 1990). L'inventaire des caractéristiques géologiques de tous ces gisements dépasse le cadre fixé à cette communication. Nous nous limiterons ici exclusivement à une présentation sommaire des gisements en Lorraine et invitons les lecteurs à consulter l'article récent des auteurs (Ghoreychi et Daupley, 2002) pour plus de renseignements sur les différents gisements de sel en France. Rappelons cependant que ces derniers appartiennent principalement à deux entités géologiques: le Trias et le Tertiaire. Seul, le Trias est présent en Lorraine et a fait l'objet de nombreuses exploitations dans les vallées de la Meurthe, du Sânon et de la Seille (Fig. 1). Il s'agit de dépôts sédimentaires organisés en couches subhorizontales, peu affectées par des mouvements tectoniques.

Plusieurs observations peuvent être faites à partir de la coupe schématique présentée sur la figure 2 (coupe oblique W-E-NE repérée sur la figure 1):

1) les gisements triasiques lorrains sont constitués de sel primaire appartenant pour l'essentiel au Keuper (Trias supérieur) et en partie au Muschelkalk (Trias moyen). Ce dernier, plus ancien que le premier, fut exploité à Sarralbe entre 200 et $300 \mathrm{~m}$ de profondeur. L'épaisseur du banc de sel gemme n'y dépasse guère une vingtaine de mètres. En revanche, le sel du Keuper est nettement plus épais et atteint une centaine de 


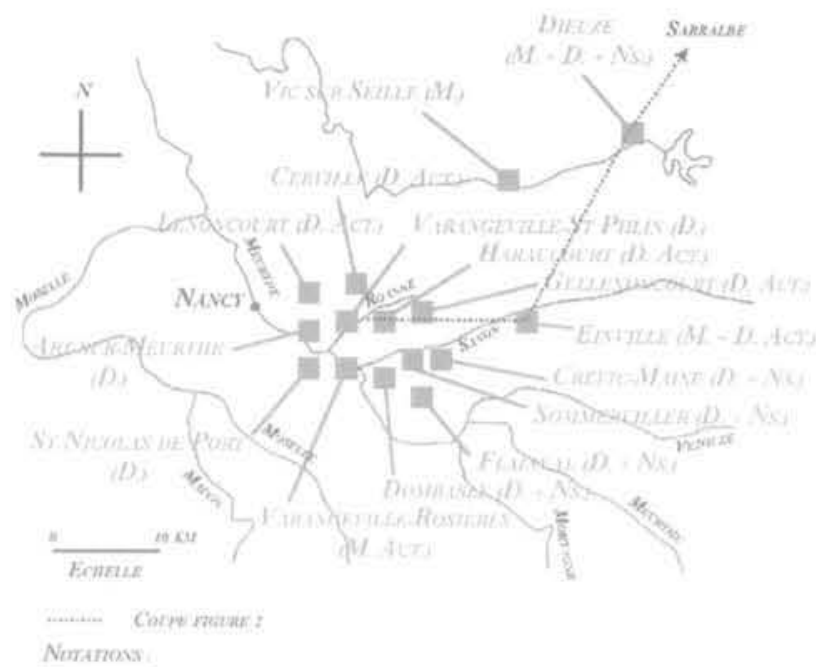

Noxamexs

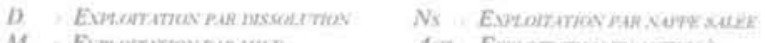

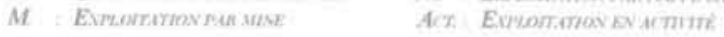

FIG. 1 Localisation des exploitations du gisement du Keuper en Lorraine (complété à partir de Marchal et al., 1994).

mètres d'épaisseur à Nancy. Cette épaisseur diminue progressivement vers le Nord-Est de sorte que le sel du Keuper n'est plus présent à Sarralbe. Ce fait témoigne d'une dissolution naturelle de ce sel au contact des eaux souterraines:

2) la présence de nappes salées a été révélée au toit immédiat du sel du Keuper. Il s'agit de phénomènes localisés rencontrés notamment dans les vallées du Sânon et de la Seille où les nappes salées sont présentes localement au toit des anciennes mines de sel d'Einville (vallée du Sânon) et de Dieuze (vallée de la Seille). Cette dernière mine fut d'ailleurs envahie par de la saumure en 1864 condamnant ainsi son exploitation. Précisons que les nappes salées furent exploitées jusqu'à présent d'une manière importante et depuis longtemps sans que cette opération, réalisée sur de très lonques périodes ne conduise à une baisse de la concentration en sel de la saumure présente dans les nappes salées. Ce fait témoigne d'une dissolution active, hypothèse compatible avec la disparition du gisement du sel du Keuper, dans le secteur de Sarralbe. L'existence d'un recouvrement peu épais associé à la présence de discontinuités mettant en communication le gisement avec des aquifères dans le recouvrement semblent être les facteurs principaux de l'apparition de ce phênomène (Marchal, 1987), Le cas de la nappe salée de Dombasle en est un bon exemple (Combes et al., 1984);

3) le recouvrement des gisements salifères lorrains est constitué essentiellement de terrains marneux, calcaires et anhydritiques. Il s'agit des faciès réputés imperméables isolant hydrauliquement l'essentiel du gisement des aquifères sous et sus-jacents. Si les terrains marneux sont dans l'ensemble déformables et peu résistants, des faciès nettement plus raides et résistants, essentiellement composés de la dolomie de Beaumont et l'anhydrite sont présents dans le recouvrement du sel du Keuper. Le banc formé par la dolomie de Beaumont et l'anhydrite n'est pas rencontré à Sarralbe et à Dieuze. Ailleurs, son épaisseur atteint au plus une dizaine de mètres. Sa profondeur varie entre zéro et une centaine de mètres dans la partie la plus profonde du gisement

4) en dehors de nappes superficielles, les niveaux plus ou moins aquifères présents au toit des gisements lorrains se trouvent dans les grès rhétiens, dans la dolomie de Beaumont, dans les calcaires du Muschelkalk et, dans une moindre mesure, dans les fissures existant dans les terrains comme les marnes irisées. II s'agit d'aquifères à faible hydrodynamisme peu ou pas exploités (le grès du Rhétien et la dolomie de Beaumont sont exploités localement); pour mémoire la transmissivité hydraulique de la dolomie de Beaumont est de l'ordre de $3 \cdot 10^{-4}$ à $3.10^{-5} \mathrm{~m}^{2} / \mathrm{s}$. Le seul niveau aquifère largement exploité correspond aux grès du Trias inférieur (gres vosgiens) qui se trouvent à une ou plusieurs centaines de mètres de profondeur, selon le contexte, au mur des terrains saliferes du Keuper et du Muschel-

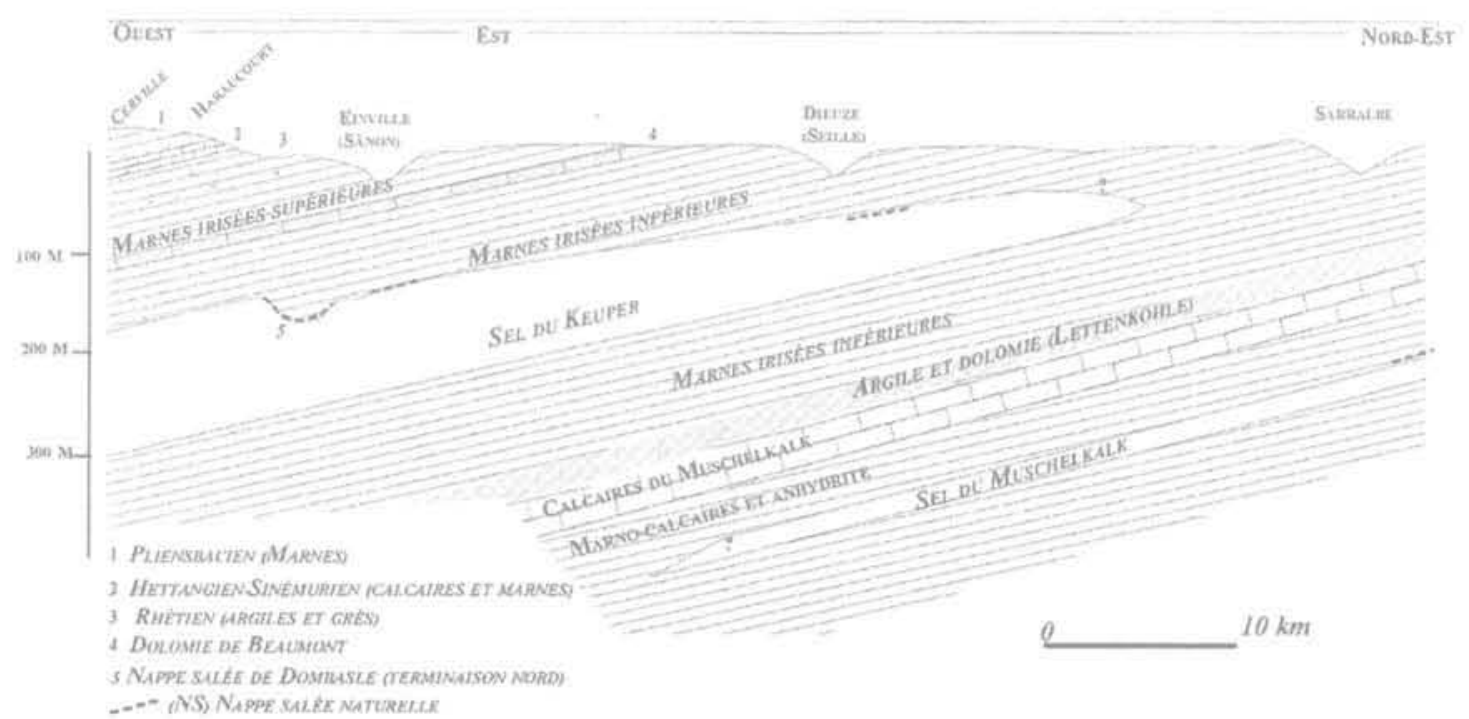

FiG. \& Coupe géologique schématique des gisements de sel lorrains passant par les principales zones d'exploitation. 
kalk. C'est un aquifére potentiellement en charge par rapport aux niveaux exploités dans le sel. Initialement artésien, sa charge hydraulique fut encore plus élevée dans le passé avant d'être affectée par une baisse significative du fait du pompage réalisé dans les différentes mines lorraines. Naturellement, un arrêt définitif du pompage, provoqué par la cessation prochaine de l'activité de nombreuses mines de la région (notamment les Houillères du bassin de Lorraine) conduirait à une surcharge hydraulique de la nappe vosgienne. Toutefois, en ce qui concerne les gisements salifères lorrains, suffisamment éloignés de ces mines, cette augmentation pourrait représenter quelques mètres.

\section{3}

\section{Caractéristiques de l'exploitation}

Si l'on exclut le seul cas d'exploitation de sel et de potasse, réalisée en France par la méthode de longues tailles aux Mines de Potasse d'Alsace, les techniques d'exploitation mises en ceuvre dans le sel en France et plus particulièrement en Lorraine peuvent être rassemblées en deux catégories:

a) la méthode d'extraction minière souterraine par chambres et piliers abandonnés. Elle a été employée dans les anciennes mines de Dieuze et d'Einville ainsi que dans la mine encore en activité de Saint-Nicolas à Varangéville;

b) les différentes techniques de dissolution avec lesquelles l'essentiel du sel continue d'être exploité (elles permettent de générer chaque année environ 2,7 millions de mètres cubes de cavités sur l'ensemble du territoire français dont la Lorraine pour une large part). Ces techniques sont nombreuses et variées. Si l'on se limite au seul cas de gisements à faible profondeur (typiquement jusqu'à 300 m), comme ceux de Lorraine, ces méthodes peuvent être divisées en plusieurs groupes:

- les techniques d'exploitation par sondages isolés au toit du sel. Celles-ci peuvent être divisées, à leur tour, en méthodes utilisant les sondages uniques et celles employant les doublets de sondages:

- les méthodes d'exploitation par sondages groupés. Elles comportent plusieurs techniques: groupe de sondages réalisés au toit du sel, exploitation partielle du gisement à partir du mur de sel par des techniques dites extensives visant à assurer la stabilité mécanique des cavités (en laissant une certaine épaisseur du sel au toit des cavités) et exploitation totale du gisement à partir du mur par des méthodes dites intensives destinées à provoquer volontairement l'effondrement du toit des cavités ayant atteint des dimensions critiques. La méthode des pistes et sondages appartient à cette dernière catégorie. Dans certaines méthodes extensives, la communication entre les sondages est assurée par le biais de la fracturation hydraulique. La méthode des pistes et sondages, quant à elle, comporte plusieurs phases: foration des sondages, formation de chenaux de dissolution mettant en communication les sondages à la base du gisement et exploitation du sel par dissolution.

On remarque que chaque type d'exploitation pose des problèmes spécifiques. Par exemple, dans une exploitation par chambres et piliers abandonnés, la stabilité mécanique du recouvrement, bien qu'en principe assurée pendant la durée de l'exploitation, peut être compromise à long terme, après l'abandon de la mine. Il va de soi que le respect de cette stabilité est nécessaire aussi bien pour garantir la sécurité des personnes et des biens en surface du sol (en particulier vis-à-vis d'un effondrement d'origine mécanique ou provoqué par l'invasion d'eau) que d'un point de vue de l'impact environnemental (notamment, le risque d'une pollution des aquifères).

Il en est de méme pour une exploitation par dissolution réalisée par une méthode extensive bien que, à la différence d'une mine sèche, les cavités de dissolution abandonnées soient remplies de saumure et communiquent déjà avec la surface du sol par l'intermédiaire de nombreux sondages d'accès.

La situation est différente pour une exploitation intensive: l'effondrement du toit faisant partie intégrante de la méthode d'exploitation, il n'y a pas lieu de craindre une nouvelle instabilité de la surface du sol. En revanche, la communication, déjà établie entre le sel et les eaux souterraines et/ou de surface (au travers le toit effondré), mérite une attention particulière.

\section{Divers aspects du comportement du sel et leurs conséquences pour les exploitations abandonnées}

\section{1}

\section{Comportement différé}

Le comportement mécanique des évaporites, en particulier le sel gemme, a fait l'objet de nombreuses recherches tant expérimentales que théoriques (Langer, 1981; Vouille et al., 1981; Munson et al., 1982; Carter et al., 1983; Spiers, 1989; Stockes, 1989; Ghoreychi, 1990). La plupart de ces recherches ont été réalisées ces dernières décennies depuis que les formations salifères sont utilisées pour y réaliser les cavités lessivées destinées à stocker les produits pétroliers, les ouvrages de stockage de déchets toxiques et les sites d'enfouissement de déchets radioactifs. Par ailleurs, le comportement du sel gemme a été étucié par la communauté de géologues et pétroliers intéressés par les phénomènes de diapirisme et de montée de dômes de sel.

L'originalité de ces études tient au fait que contrairement à la plupart des roches, les mécanismes microscopiques responsables de la déformation de sel sont bien connus et intégrés dans les modèles rhéologiques. Il est unanimement admis que les déformations des cristaux de halite ( $\mathrm{NaCl}$ ) peuvent être de trois origines: mouvements de dislocations (montées et glissement), diffusion et solution-précipitation. Ces mécanismes se traduisent par un comportement rhéologique macroscopique bien identifié, celui représenté par le corps viscoplastique de Maxwell, dépourvu de tout seuil d'écoulement différé. Ce fait est conforme aux résultats d'essais de fluage réalisés sur le sel: les déformations différées (se produisant dans le temps) apparaissent quel que soit le niveau de contrainte déviatorique appliquée. L'amplitude de ces dernières dépend fortement de ce déviateur: elle peut être très importante sous forte sollicitation ou devenir négligeable, voire imperceptible, si le déviateur est très faible (situation rencon- 
trée loin des ouvrages souterrains). Néanmoins, le sel continue à fluer lentement tant qu'il est soumis à une contrainte déviatorique, même très faible. Ce phénomène se poursuit sans cesse à moins que la roche soit soumise à un état de contrainte isotrope (état naturel d'un massif de sel au repos).

Dans toutes les lois rhéologiques proposées pour le sel, la relation entre la vitesse de fluage et la contrainte déviatorique est exprimée par une loi de puissance. En outre, l'aptitude au fluage du sel augmente considérablement avec la température. Ce comportement est exprimé classiquement par une lol exponentielle dite d'Arrhenius, fréquemment employée en thermodynamique.

Au cours d'un essai de fluage, réalisé sous contrainte déviatorique $\left(\sigma_{f}\right)$ et température $(\mathrm{T})$, la vitesse de déformation du sel diminue d'abord pendant une phase transitoire appelée « fluage primaire » avant de se maintenir sensiblement constante pendant une deuxième phase qualifiée de fluage stationnaire, établi ou encore secondaire (de l'anglais steady-state creep). Pour reproduire ces résultats, deux lois rhéologiques ont été proposées:

- la loi de Norton (ou Norton Hoff) considérant une stabilisation de la vitesse de fluage $\left(\varepsilon^{\prime}\right)$ au-delà d'une phase transitoire: $\varepsilon=a(\sigma)^{n}$. Cette loi est largement utilisée pour le sel à travers le monde (Langer, 1981; Wawersik et al., 1981; Munson et al., 1982; Spiers, 1989);

- la loi baptisée Lemaitre (en France) ou Menzel-Schreiner (en Allemagne). Elle considère que la vitesse de fluage du sel diminue dans le temps mais très lentement. Ce qui revient à admettre, en termes rhéologiques, l'existence d'un écrouissage en déformation (cela exprime que le matériau devient de plus en plus raide en se déformant; ce concept est contesté par la loi de Norton). Ce type de loi ne fait aucune distinction entre les fluages primaire et secondaire et exprime les résultats d'un essai de fluage à l'aide une fonction puissance de temps $\left(\varepsilon=a t^{\alpha}: \varepsilon\right.$ et $t$ : déformation et temps respectivement). Plus précisément, la vitesse de déformation viscoplastique $(\varepsilon)$ est exprimée par la relation suivante: $\varepsilon^{*}=\mathrm{a}\left(\sigma_{f}\right)^{\mathrm{n}} \varepsilon^{\mathrm{m}}$. Cette loi rhéologique est moins répandue que celle de Norton. Elle est utilisée exclusivement en France (proposée et largement utilisée par l'École des mines de Paris, (Vouille et al., 1981) et dans une moindre mesure, en Allemagne (uniquement en ex-Allemagne de l'Est).

En dépit des controverses qui opposent les partisans et les adversaires de ces lois, les deux modèles s'accordent, pour l'essentiel, sur les principaux traits du comportement rhéologique du sel gemme: comportement viscoplastique dépourvu de tout seuil de fluage, dépendance de ce comportement vis-à-vis de la seule partie déviatorique du tenseur de contraintes, caractère standard des lois proposées (lois associées : critère et potentiel de viscoplasticité identiques), etc., autant d'aspects, importants d'un point de vue rhéologique, qui sont communs aux deux modèles. Quant à l'existence ou non d'un fluage stationnaire, à notre avis, sa vérification n'est pas du ressort d'expérience de laboratoire. En effet, les résultats d'un essai de fluage peuvent être interprétés différemment suivant que l'on réactualise ou non l'aire de la section d'un échantillon, déformée pendant son fluage. Ce problème se pose également pour l'interprétation des mesures relatives de convergence et d'extensométrie, réalisées sur les ouvrages dans le sel. Précisons cependant que toutes les mesures de déplacements et de déformations, effec- tuées dans le sel à travers le monde sur de nombreux ouvrages souterrains, indiquent un comportement pratiquement stationnaire. Ce type de comportement est observé quelques mois à quelques années après le creusement de l'ouvrage, selon les dimensions et la profondeur de celui-ci. De ce fait, le concept de fluage établi semble être bien vérifié in situ. Il n'en demeure pas moins que les mesures in situ peuvent être reproduites aussi bien par le modèle de Norton que par celui de Lemaitre (qui n'admet pas le fluage stationnaire). Les résultats des études réalisées avec ce dernier modèle par l'École des mines de Paris sur de nombreuses mines et cavernes de stockage dans le sel en sont une illustration convaincante (Vouille et al., 1981).

Compte tenu de l'ensemble de ces considérations, nous pensons que la loi de Norton tout comme le modèle rhéologique de Lemaitre peuvent être utilisés pour les études à long terme des exploitations de sel. L'essentiel est le jeu de paramètres introduits dans l'un ou l'autre de ces modèles plutòt que leur expression mathématique. L'expérience prouve que, quelle que soit la variété de sel, son comportement différé peut ètre décrit convenablement à l'aide des modèles mentionnés. Cependant, les valeurs des paramètres y intervenant sont susceptibles de varier considérablement selon le sel, voire au sein d'une même formation géologique. Ce fait suggère que la détermination des valeurs des paramètres rhéologiques de chaque sel fasse l'objet d'une caractérisation spécifique. Pour cela, il convient de réaliser une série d'essais de fluage avec paliers multiples de contrainte déviatorique sous une température proche de celle du massif. Le jeu de paramètres ainsi déterminés doit être ajusté, autant que possible, par des mesures in situ réalisées sur l'ouvrage étudié. Cet ajustement permettrait d'intégrer l'effet de la variation spatiale des caractéristiques rhéologiques du sel. bien que ce matériau ne soit pas trop affecté par des discontinuités comme c'est souvent le cas d'autres roches (la grande ductilité du sel fait diminuer considérablement l'effet d'échelle lié aux discontinuités).

Par ailleurs, il nous paraît important que les codes de calcul destinés à l'étude du comportement à long terme des exploitations de sel intègrent aussi bien la loi de Norton que celle de Lemaitre. L'implantation de ce dernier modèle serait suffisante puisque la loi de Norton est un cas particulier du modèle de Lemaitre pour lequel la valeur de l'exposant de la déformation viscoplastique est nulle $(\mathrm{m}=0)$.

Pour mémoire, la généralisation à trois dimensions de la loi viscoplastique du sel susceptible d'être introduite dans les codes de calcul s'écrit sous la forme tensorielle suivante:

$$
\left.\partial \varepsilon_{i j} p / \partial t=A \exp (-B / T)\left(\sigma_{e q} / \sigma_{0}\right)^{n}\left(\varepsilon^{v p}\right)^{m}\right)^{m} \partial s_{e q} \partial \sigma_{i}
$$

$\sigma_{e q}$ : contrainte déviatorique équivalente liêe au deuxième invariant du tenseur déviateur de contraintes Sij; $\sigma_{\mathrm{ecl}}=(3 / 2 \text { Sij: } \mathrm{Sij})^{1 / 2}$;

$\varepsilon^{\mathrm{vp}}$ : déformation viscoplastique équivalente; $\varepsilon^{\mathrm{vp}}{ }_{\mathrm{eq}}=$

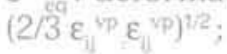

$\sigma_{j}$ et $\varepsilon_{i j}$ : tenseurs de contrainte et de déformation. viscoplastique;

$\partial \varepsilon_{i j}{ }^{v p} / \partial t$; tenseur vitesse de déformation viscoplastique (son unité est l'inverse du temps);

$\sigma_{0}$ contrainte de référence $\sigma_{0}=1 \mathrm{MPa}$;

$\mathrm{T}$ : température (Kelvin);

A, B, n et $m$ : paramètres du modèle ( $m=0$ pour la loi de Norton). 


\section{Remarques}

1) La valeur de l'exposant de contrainte varie en général entre 3 et 5, selon le sel. Cela signifie que, toutes choses identiques par ailleurs, si la profondeur d'un ouvrage dans le sel est doublée, la vitesse de convergence de celui-ci peut être multipliée par un facteur compris entre 8 et 32 . Par ailleurs, la valeur du paramètre B (lié à l'énergie d'activation, dans la loi d'Arrhenius) est généralement comprise entre 3000 et 7000 (en degré Kelvin). Par conséquent, une variation de température du massif de $10^{\circ} \mathrm{C}$ entre 20 et $30^{\circ} \mathrm{C}$ peut conduire à une multiplication de la vitesse de fluage du sel par un facteur compris entre 1,4 et 2,2. Ces exemples montrent la grande sensibilité et la forte non-linéarité du fluage du sel vis-à-vis de l'état de contrainte et de la température.

2) Assimiler le comportement différé du sel à celui d'un fluide visqueux ne signifie pas nécessairement que tout ouvrage réalisé en son sein se referme sur luimême à court ou à moyen terme. Cela dépend essentiellement de la profondeur de l'ouvrage, mais d'autres facteurs comme la forme et les dimensions de l'ouvrage et la nature des terrains non salifères interviennent également. Pour mémoire, la vitesse des déformations différées des piliers des mines de sel en Lorraine est typiquement de quelques $\mathrm{mm} / \mathrm{an}$ ce qui est évidemment très faible et n'est pas de nature à conduire à une fermeture des vides sur quelques siècles.

3) Le comportement du sel gemme étant èlastoviscoplastique, ses propriétés élastiques peuvent également jouer un rôle important. Ce fait tient en particulier à la valeur relativement élevée du module de Young du sel (environ $25 \mathrm{GPa}$ ) conférant à ce matériau une rajdeur importante en flexion. Cet aspect revèt une grande importance pour la tenue mécanique du banc de sel laissé au toit de certaines cavités de dissolution ou mines de sel. La présence de ce banc est un élément très favorable à la stabilité du toit de ces exploitations. Lorsque le sel n'est pas présent au toit d'une exploitation, les terrains argileux se trouvant au toit du sel se détachent des terrains sus-jacents et tombent progressivement dans les cavités ou les chambres sous leur propre poids. Cette situation peut conduire, en Lorraine, à une sollicitation excessive des bancs raides d'anhydrite et de dolomie de Beaumont. Leur rupture est de nature à conduire à l'effondrement du recouvrement, comme cela se produit volontairement dans la méthode de dissolution intensive. Un tel scénario doit être évité dans la méthode de dissolution extensive de même que pour les exploitations par chambres et piliers. Pour cela, la présence d'un banc de sel d'épaisseur suffisante au toit de cavités est indispensable fauté de quoi (aux bordures du gisement ou lorsque l'exploitation progresse trop au toit de sel) la stabilité à long terme de l'exploitation ne peut plus être assurée.

\section{2}

\section{Endommagement et rupture}

Par rapport à la viscoplasticité, l'initiation et le développement de la fissuration du sel ont été relativement peu étudiés. Ce fait tient à la grande ductilité de ce matériau qui lui confère une aptitude à subir des grandes déformations sans se rompre nécessairement. C'est en particulier le cas lorsque le sel est soumis à une contrainte moyenne de compression dépassant une dizaine de $\mathrm{MPa}$. Aucun pic de résistance, révélateur d'une rupture macroscopique, n'est atteint au cours d'un essai de compression triaxiale, réalisé sous une pression de confinement supérieure à environ $10 \mathrm{MPa}$. Pour des valeurs de pression plus faibles, la rupture apparait lorsque le sel est soumis à des vitesses de déformation relativement importantes (Wallner, 1983; Hunche, 1993), comme ce peut être le cas à court terme autour des ouvrages souterrains. C'est ainsi qu'est expliquée l'accélération brutale de la vitesse de déformation (fluage tertiaire), constatée en laboratoire, conduisant à la rupture d'échantillons soumis aux essais de fluage. Notons en outre que la rupture du sel intervient également en traction, comme c'est le cas de toutes les roches.

Des études expérimentales approfondies débouchant sur des lois d'endommagement et de rupture de sel furent réalisées, notamment au cours de ces dix dernières années (Cristescu et al., 1991; Thorel, 1994; Thorel et al., 1996). Ces études portent essentiellement sur le comportement à court terme du sel. Leurs résultats suggèrent que ce matériau ne puisse subir une rupture différée à moins qu'il soit déjà endommagé à court terme par suite d'une sollicitation dépassant son seuil d'endommagement (Thorel, 1994). Cé résultat obtenu en laboratoire est à vérifier in situ.

Deux critères sont déterminés expérimentalement: - celui exprimant l'initiation d'endommagement, déterminée à partir de la límite de linéarité de la courbe «contrainte moyenne-déformation volumique irréversible ». L'initiation de l'endommagement se traduit par la manifestation d'une dilatance (augmentation de volume), Cet indicateur se justifie par le fait que la déformation viscoplastique du sel intact (non endommagé) se produit sans aucune variation de volume; - le critère de rupture déterminé à partir de valeurs correspondant au pic de résistance, mesurées par des essais triaxiaux.

Ces critères font intervenir trois scalaires liés aux tenseurs de contraintes et de leurs déviateurs: la contrainte moyenne, la contrainte déviatorique équivalente (de Von Mises) et la géométrie de contrainte (liée à l'angle de Lode). Ce dernier paramètre permet d'exprimer le fait que l'endommagement de sel est plus précoce en extension qu'en compression. Dans les deux cas, le critère d'endommagement et celui de rupture sont approximés par des expressions paraboliques, données dans le plan «contrainte moyennecontrainte déviatorique $\pi$. Ainsi, à la différence du comportement différé qui est indépendant de la contrainte moyenne et qui apparait quel que soit le déviateur de contraintes, l'endommagement et la rupture du sel n'interviennent qu'au-delà d'un certain seuil fonction de la contrainte moyenne. En d'autres termes, le fluage du sel est indépendant du frottement interne de ce matériau alors que ce dernier est bien mobilisé par les processus d'endommagement (microfissuration) et de rupture (fracturation).

A titre indicatif, les valeurs des seuils d'endommagement et de rupture, obtenues en compression uniaxiale varient en général, selon le sel, dans les intervalles suivants:

- seuil d'endommagement: 8 à $12 \mathrm{MPa}$;

- résistance à la rupture: 18 à $30 \mathrm{MPa}$.

Pour des cavités souterraines, les risques d'endommagement et de rupture du sel doivent être évalués en fonction de la profondeur (liée à l'état de contraintes ini- 
tiales isotropes résultant du poids du recouvrement), de la géométrie de l'ouvrage et de ses dimensions. Le recours à une modélisation numérique (par exemple, aux éléments finis) s'impose dans la plupart des cas, le calcul analytique ne donnant qu'une première indication approchée de l'état de stabilité. Par exemple, pour une exploitation par chambres et piliers abandonnés, la valeur moyenne de la contrainte verticale régnant dans les piliers, donnée par le calcul classique d'équilibre d'aire tributaire peut être surestimée si un banc épais de sel est laissé au toit. Celui-ci peut participer à l'équilibre global de l'exploitation et soulager, en partie, les piliers et ce d'autant plus que la largeur totale de l'exploitation est faible (limitation par des stots ou des bords fermes). Par ailleurs, l'existence de contraintes horizontales dans la partie centrale des piliers (en particulier pour des piliers à faible élancement) est un facteur favorable à la stabilité de ces derniers et contribue également à réduire leur déformation au fluage (par le biais d'une diminution de la contrainte déviatorique). Comparer la valeur de la contrainte verticale calculée par l'aire tributaire à celle de la résistance uniaxiale correspondant à l'endommagement ou à la rupture condulrait, là encore, à une évaluation trop pessimiste de la stabilité de l'exploitation. Ce fait est confirmé par de nombreuses mines de sel en France et à l'étranger dont la stabilité mécanique est assurée depuis plus d'un siècle sans que cet état de fait ne puisse être expliqué par l'analyse classique simplifiée qui vient d'être évoquée. La stabilité des piliers des anciens quartiers de la mine de Varangéville, exploités dans le passé avec un taux de défruitement de $75 \%$, et celle de la mine de Dieuze dont le taux de défruitement avoisine localement la valeur très élevée de $90 \%$, en sont des bons exemples.

\section{3}

\section{Influence des fluides}

Les résultats des essais de laboratoire attestent que les caractéristiques viscoplastiques, d'endommagement et de rupture du sel sont sensiblement modifiées au contact de l'humidité ou de la saumure (même parfaitement saturée). La résistance mécanique du sel diminue alors que son aptitude au fluage augmente. Ce phénomène s'explique par la conjugaison de plusieurs facteurs (Lai, 1971; Brodsky et al., 1991; Peach, 1991; Fokker et al., 1993; Cosenza et al., 1997):

- l'accélération de mouvements de dislocations en présence de la saumure contribuant à la diminution de la viscosité du sel:

- l'activation du phénomène de solution-précipitation sous contrainte (de l'anglais pressure solution);

- l'effet de la pression interstitielle de saumure s'introduisant dans les microfissures,

En raison de la grande solubilité du sel gemme dans l'eau, on conçoit que la stabilité d'une exploitation de sel envahie par de l'eau douce puisse être facilement compromise. Ce scénario accidentel s'est déjà produit dans de nombreuses mines de sel et de potasse dans le monde et ne peut être exclu dans certains cas en France.

Pour les gisements lorrains, nous avons vu que les niveaux aquifères présents au toit ne sont pas très importants et sont séparés du sel par un faciès marneux très peu perméable. En revanche, des nappes salées sont directement en contact avec le sel au toit de certaines exploitations et soulèvent le problème de l'invasion à long terme éventuelle des mines concernées, par de la saumure saturée. On peut alors s'interroger sur les conséquences d'une telle éventualité sur la stabilité mécanique de ces mines. C'est exclusivement sur cette éventualité que nous allons nous baser dans la suite plutôt que sur les conséquences évidentes d'une invasion d'eau douce.

Pour apprécier l'influence des fluides a priori non réactifs, notamment la saumure saturée, sur les propriétés de résistance du sel, nous nous référons aux résultats de quelques expériences réalisées il y a quelques années, à l'École polytechnique (Cosenza, 1996; Cosenza et al., 1999). La figure 3 rassemble ces. résultats.
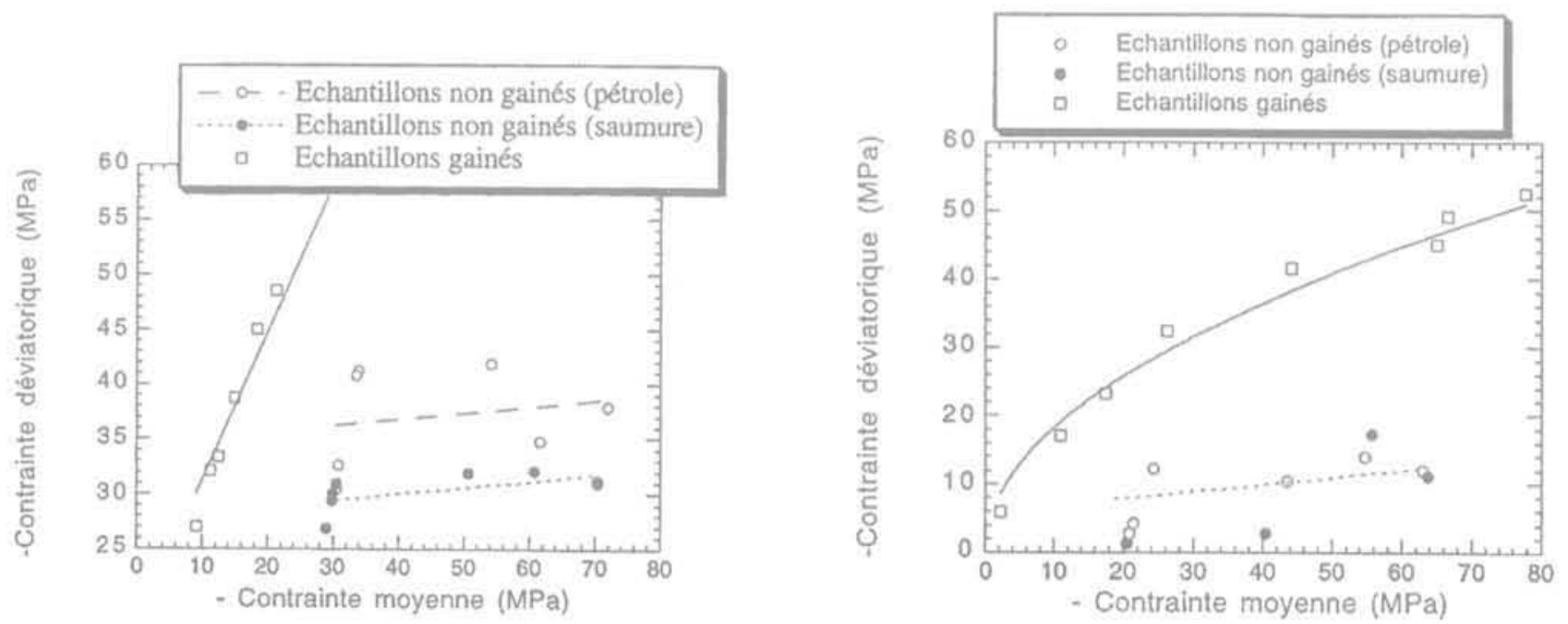

FIG. 3 Comparaison des critères de rupture (à gauche) et d'endommagement (à droite) d'échantillons de sel placés ou non au contact d'un fluide (essais réalisés sur le sel gemme provenant des mines de potasse d'Alsace, Cosenza, 1996). 
Deux types d'expériences ont été effectués:

- des essais triaxiaux conventionnels, effectués sur les échantillons couverts d'une gaine étanche et placés, de ce fait, à l'abri de tout contact avec le fluide de confinement;

- des essais réalisés dans les mêmes conditions de sollicitation mais en retirant, cette fois, la gaine protectrice pour mettre volontairement les échantillons au contact direct d'un fluide sous pression.

En outre, deux types de fluide ont été utilisés pour la deuxième série d'essais, effectués sur les éprouvettes non gainées: une saumure parfaitement saturée et du pétrole, a priori sans interaction chimique avec le sel.

On remarque que les valeurs de la résistance correspondant à l'endommagement ou à la rupture, obtenues sur les échantillons soumis au contact d'un fluide sous forte pression sont bien inférieures à celles des échantillons placés à l'abri de ce contact. Cette différence est d'autant plus importante que la pression de fluide exercée directement sur le sel est élevée. Tout se passe comme si le sel perdait l'essentiel de sa capacité à mobiliser son frottement interne, en présence d'un fluide. A titre indicatif, l'angle de frottement interne déterminé sur les échantillons non gainés est de l'ordre de $10^{\circ}$ alors que celui des échantillons gainés est entre 30 et $40^{\circ}$. En revanche, la valeur de la cohésion ne semble pas trop varier en contact d'un fluide. Autrement dit, la diminution de la résistance est très significative notamment sous forte pression de fluide.

Pour une valeur de pression de fluide donnée, la résistance à la rupture est plus faible en présence de la saumure qu'au contact du pétrole. Mais la différence n'est pas très significative en ce qui concerne l'initiation de l'endommagement. Ce résultat suggère que la baisse de la résistance soit attribuée davantage à une action mécanique de la pression de fluide qu'à une interaction chimique entre le sel et la saumure. Cette hypothèse semble plausible dans la mesure où la saumure utilisée était parfaitement saturée avec le même sel que celui constituant les échantillons. Aucune altération chimique du matériau pouvant occasionner une chute drastique de sa résistance mécanique ne pouvait donc se produire pendant les quelques heures qu'a duré un essai. Pourtant, tous les échantillons de sel soumis au contact d'un fluide ont subi une rupture fragile, semblable à celle observée en l'absence de toute pression de confinement (compression uniaxiale). Cette fragilité a été systématique pour tous les échantillons non gainés et ce quelle que soit la pression de fluide appliquée. Ce résultat a èté confirmé avec une valeur de pression de fluide particulièrement élevée, de $60 \mathrm{MPa}$, appliquée à quelques échantillons. La rupture du sel soumis au contact d'un fluide demeure aussi fragile à $60 \mathrm{MPa}$ qu'en compression uniaxiale ! Ce résultat parait d'autant plus surprenant que le comportement du sel placé à l'abri de tout fluide demeure, comme nous l'avons déjà précisé, parfaitement ductile au-delà seulement de $10 \mathrm{MPa}$ de pression de confinement.

A notre avis, cette différence de comportement, très significative, ne peut ètre expliquée que par le développement d'une pression interstitielle atteignant le coeur des échantillons pendant les essais. Cela reviendrait à admettre que les échantillons étaient initialement quelque peu microfissurés et que cet endommagement a atteint leur cœur. Pourtant, la valeur de la perméabilité intrinsèque initiale des échantillons testés, mesurée avant chaque essai était systématiquement inférieure à
$10^{-19} \mathrm{~m}^{2}$ (équivalent de $10^{-12} \mathrm{~m} / \mathrm{s}$, à l'eau) ce qui est négligeable et conforme aux ordres de grandeurs habituelles de la perméabilité de sel, mesurée aussi bien au laboratoire qu'in situ $\left(10^{-21}-10^{-19} \mathrm{~m}^{2}\right)$. Ces valeurs, typiques des milieux réputés imperméables selon les hydrogéologues, sont néanmoins suffisantes pour permettre, pendant la durée d'un essai, la percolation des fluides utilisés au cœur de l'échantillon. Ce fait tient à plusieurs facteurs: le petit diamètre des échantillons $160 \mathrm{~mm}$, diamètre non négligeable pour un échantillon de laboratoire mais classique pour le sel du fait de sa taille de grains), la pression élevée du fluide, de quelques dizaines de MPa (plus précisément, le gradient de pression élevé, imposé entre le bord et le cœur de l'échantillon) et la perméabilité (très faible mais non nulle) des échantillons.

Ces résultats suggèrent que le contact direct entre le sel et la saumure soit un facteur défavorable à la stabilité mécanique, du moins à l'échelle du laboratoire. En est-il de même in situ pour les cavités dans le sel remplies volontairement ou accidentellement de saumure?

Il serait imprudent de transposer directement, et d'une manière trop rapide, les résultats obtenus sur les échantillons de laboratoire au cas d'un massif rocheux encaissant les cavités souterraines. En effet, pour une cavité de dissolution isolée par exemple, le fluide n'entoure pas la roche comme c'est le cas d'un échantillon de laboratoire immergé. On conçoit que, dans l'ensemble, le massif encaissant la cavité se trouve dans un état plus proche de celui d'un échantillon sec que de l'état d'une éprouvette baignant dans un fluide sous pression. Cette hypothèse est confirmée par le fait que les lois rhéologiques caractérisées par des essais de fluage, qui sont réalisés habituellement sur les échantillons secs, reproduisent assez bien les déformations mesurées sur les cavités de dissolution (il s'agit de cavernes destinées au stockage de produits pétroliers sur lesquelles ce type d'études a souvent été réalisé nous ignorons si c'est aussi le cas de cavités d'exploitation de sel par dissolution).

La situation est différente pour une exploitation par chambres et piliers abandonnés, soumise à une invasion accidentelle de la saumure provenant de nappes salées, Dans ce cas, l'analogie entre l'échantillon de laboratoire et le pilier aurait plus de sens. Encore faudrait-il tenir compte de l'effet d'échelle, très important dans cette comparaison. L'éprouvette de laboratoire étant nettement plus petite que le pilier, le fluide atteint beaucoup plus rapidement son coeur que celui du pilier. En effet, toutes choses identiques par ailleurs, le temps nécessaire à la percolation du fluide est proportionnel au carré de distance parcourue. En considérant qu'un pilier d'une mine de sel est en général au moins 100 fois plus grand qu'un échantillon de laboratoire, on peut estimer que le temps nécessaire à la percolation de saumure dans un pilier peut être au moins de quatre ordres de grandeur supérieur à celui d'un échantillon de laboratoire. Ce temps peut être de quelques années pour un pilier de $10 \mathrm{~m}$ de largeur soumis aux mêmes conditions de perméabilité et de pression que les échantillons de laboratoire. Cela indique que l'action mécanique de la saumure ne peut pas être rapide in situ tant que la perméabilité du sel n'est pas trop altérée par une fissuration.

Dans la pratique, le temps ainsi estimé peut changer considérablement en fonction des valeurs de la perméa- 
bilité du pilier et de la pression du fluide (le temps est proportionnel à ces dernières et la perméabilité est susceptible de varier considérablement au sein d'un pilier).

A notre connaissance, aucune mesure in situ d'évolution spatiale de la perméabilité n'a été réalisée jusqu'à présent dans un pilier d'une mine de sel. On ignore donc si le cœur du pilier peut être effectivement étanche à la saumure ou s'il peut présenter, au contraire, une certaine perméabilité, même très faible. Aussi, il peut être utile de se référer aux résultats obtenus sur d'autres ouvrages dans le sel. De ce point de vue, les résultats des mesures in situ de perméabilité, effectuées autour des galeries ou des forages sur le site salifère de WIPP (Waste Isolation Power Plant) au Nouveau Mexique, Etats-Unis (Stormont et al.. 1991) nous semblent intéressants. Ces mesures mettent en évidence une augmentation substantielle de la perméabilité au sein d'une zone ètendue à un à deux rayons de l'ouvrage. En particulier, la perméabilité de la paroi est de 5 à 6 ordres de grandeur supérieure à celle du massif intact situé loin de la paroi. Pourtant, ce phénomène ne se traduit par aucune fissuration visible de sel en paroi et indique clairement qu'une microfissuration. bien qu'invisible à l'oeil nu, peut être responsable d'une augmentation drastique de la perméabilité du sel.

Il est tout à fait probable que la perméabilité soit également très élevée en paroi des piliers de toute mine de sel sans que ce phénomène ne s'étende obligatoirement au cœur des piliers. Dans l'hypothèse de l'existence d'une zone centrale véritablement imperméable, la pression exercée par le fluide ne pourrait être assimilée à une pression interstitielle. Au contraire, elle agirait plutôt comme une pression de soutènement jouant un rôle bénéfique sur la stabilité du cœur du pilier. La diminution de la viscosité du sel en contact avec la saumure pourrait alors être compensée, en partie ou entièrement, par la diminution du déviateur de contraintes régnant dans le pilier, ce qui réduirait, leur tassement lié au fluage.

Ce scénario n'a de sens que si la perméabilité du cour du pilier est effectivement négligeable; il n'est pas plausible si sa valeur est faible: cette dernière situation autoriserait, sur de long terme, une introduction de la saumure au cour de piliers. Ce phénomène, sans doute très lent (selon la perméabilité et la pression de saumure) et de faible amplitude (la porosité de microfissures étant négligeable), donnerait lieu au développement d'une pression interstitielle qui serait en équilibre avec celle de la saumure présente dans les chambres. Cela réduirait, à son tour, la stabilité mécanique à long terme des piliers et plus généralement celle de l'exploitation.

Malheureusement, nous ne disposons pas d'assez de retours d'expériences sur les mines de sel envahies par de l'eau douce ou de l'eau salée, bien que de nombreuses mines de sel ou de potasse à travers le monde aient été affectées par une invasion accidentelle de l'eau. Les exemples disponibles cités dans la littérature ne sont pas suffisamment renseignés pour en dégager des conclusions convaincantes quant à l'action mécanique de la saumure sur la stabilité à long terme des exploitations de sel, en général. Dans bien des cas, les mines de sel ont été envahies par de l'eau douce dont les conséquences ne sont pas tout à fait comparables (du moins à court terme avant que l'eau ne se sature en sel) à celles d'une saumure saturée. Or, pour des raisons déjà évoquées, c'est le scénario accidentel d'une invasion de la saumure provenant de nappes salées qu'il convient d'analyser, en premier lieu, dans le contexte de l'abandon des mines de sel en Lorraine. En effet, il n'est pas exclu que ce type d'accident, qui s'est déjà produit dans le passé dans la mine de Dieuze, puisse se reproduire dans d'autres mines en Lorraine.

Les investigations in situ réalisées récemment par GEODERIS (dans le prolongement des études menées par l'TNERIS et l'École des mines de Paris sur la stabilité de cette mine) ont mis en évidence que la stabilité mécanique de la mine de Dieuze est actuellement assurée alors que plus d'un siècle s'est écoulé depuis que les nappes salées ont envahi cette mine.

A ce sujet, les résultats de la modélisation effectuée par l'École des mines de Paris sont en parfait accord avec les informations tirées des archives sur le fait que la stabilité de la mine de Dieuze était bien assurée avant son invasion. Ce fait a été expliqué (par l'École des mines de Paris) en particulier par la participation active du toit à l'équilibre général de cette exploitation. Ce phénomène tient à deux facteurs: l'épaisseur importante du sel laissé au toit (environ 50 mètres) et la largeur limitée de l'exploitation, comparée à la profondeur. Dans cette condition, avant l'invasion de la mine, le cceur des piliers n'avait très probablement pas été affecté par un endommagement lui conférant une per. méable à la saumure importante.

Il ne serait pas prudent d'étendre cette situation, spécifique à la mine de Dieuze, à l'ensemble des mines de sel, dans l'hypothèse de leur invasion éventuelle par de la saumure saturée provenant de nappes salées. Chaque cas est en effet spécifique et l'action mécanique de la saumure sur la stabilité d'exploitations de sel est encore loin d'être bien comprise et facile à maitriser. Des recherches expérimentales in situ, complétées par une analyse approfondie de l'interaction entre la saumure et les roches encaissant les exploitations en Lorraine (en particulier le sel et les argilites présents au mur de la $11^{\circ}$ couche de sel exploitée dans la région permettraient d'étudier, d'une manière appropriée cette question qui revêt une grande importance pour le devenir à long terme de ces exploitations.

\section{5}

\section{Conclusion}

L'abandon progressif des mines et des exploitations par dissolution de sel soulève la question de l'évolution à long terme des sites concernés, d'un point de vue de la stabilité mécanique de la surface du sol et de l'impact environnemental. Cette question doit être analysée pour chaque site spécifique compte tenu de plusieurs facteurs importants:

- le contexte géologique du site au sens large du terme, en prenant en considération la lithologie, la stratigraphie, la minéralogie, la tectonique...

- le contexte hydogéologique du site: présence de niveaux aquifères, leur hydrodynamisme, existence et nature de nappes salées, possibilité d'une communication à long terme entre le sel, les aquifères et les nappes salées...;

- les divers aspects du comportement spécifique du sel: fluage, endommagement, perméabilité, influence de fluides (eau, saumure, gaz s'il y a lieu)...;

- les conséquences de l'ensemble des facteurs ci-dessus sur l'évolution à long terme du site étudié (étude d'impact), 
Beaucoup de ces aspects ont déjà fait l'objet d'investigations importantes. C'est par exemple le cas de la loi de comportement différé du sel. Toutefois, certains aspects demandent encore des études complémentaires. Celles-ci devront être réalisées, de préférence, dans les domaines suivants:

1) méthodes et movens de reconnaissance de la présence et de l'état de stabilité de cavités de dissolution et de leurs forages d'accès;

2) inventaire synthétique d'instabilités mécaniques rencontrées en France et à l'êtranger dans les mines de sel traditionnelles et dans les exploitations par dissolution. Ce recensement permettrait de mettre à profit les retours d'expériences disponibles pour valider les approches mises en ceuvre pour l'étude de la stabilité à long terme d'exploitations abandonnées de sel;

3) investigation in situ de l'action mécanique de la saumure sur le comportement d'une mine de sel et l'étude approfondie des phénomènes associés (évolution de la perméabilité dans les piliers en fonction de l'état d'endommagement, phénomènes hydro-géochimiques liés à l'interaction "sel-saumure " et s'il y a lieu ("argilite-saumure », etc.);

4) adaptation des méthodologies et des moyens de l'étude de l'après-mine au contexte spécifique de sel en tenant compte de deux spécificités majeures de ce matériau: son aptitude au fluage et sa grande solubilité dans l'eau. De ce fait, les méthodologies et les moyens mis en ceuvre pour l'analyse de la fermeture et de la post-fermeture des mines traditionnelles (fer, houillères, mines métalliques, etc.) doivent être adaptés au contexte particulier des exploitations de sel quelle que soit la méthode d'extraction employée (chambres et piliers, longues tailles, dissolution);

5) pour chaque site étudié (étude spécifique d'une exploitation), investigations approfondies sur les archives disponibles (analyse informative), complétées par des études de terrain (géologie, hydrogéologie, sondages avec diagraphie et carottage des niveaux représentatifs), des études de laboratoire (essais spécifiques de caractérisation, modélisation) et des investigations in situ (auscultation et surveillance).

Étant donné le caractère clairement pluridisciplinaire de l'étude de la post-fermeture d'exploitations de sel, les travaux ci-dessus devront mettre à profit les résultats de nombreuses recherches réalisées en France et à l'étranger sur le sel et sur les ouvrages en milieux salifères (notamment dans le domaine du stockage) tout en s'appuyant sur l'expérience déjà acquise sur la fermeture et la post-fermeture des mines traditionnelles.

\section{Bibliographie}

Blanc-Valleron M.M. - Les formations paléogènes évaporitiques du bassin potassique de Mulhouse et des bassins plus septentrionaux d'Alsace. Thèse de doctorat és Sciences naturelles; université Louis-Pasteur. Strasbourg, 1990.

Brodsky N.S., Murison D.E. - The effect of brine on the creep of WIPP salt in laboratory tests, Rock Mechanics as a multidisciplinary science, Proc. 32nd US Svmp. Rock Mech. J.C. Roggiers (ed.) Balkema, 1991

Carter N.L., Hansen F.D. - Creep of rocksalt. Tectonophysics. vol. 92, 1983, p. 275333.

Combes P., Ledoux E., Marsily (de) G. Etude des dissolutions de sel en couche. CR de la fournée sur sel, $1^{\text {er }}$ mars, L.M.S. Ecole polvtechnique, Palaiseau. 1984.

Cosenza P. - Sur les couplages entre comportement mécanique et processus de transfert de masse dans le sel gemme. These de doctorat, université Paris VI, 28 octobre 1996.

Cosenza P., Ghoreychi M. - Effect of fluidrock interaction on mechanical behvior of rock sait. 5th Conf. Mechanical Behavior of Salt, Bucharest (Romania), August 9-11, Proc. Basic \& Applied Salt Mechanics, Cristecu et al. (eds), AA. Balkema, 1999, p. 57-72.

Cristescu N.. Hunsche U. - A constitutive equation for salt. 7 th Int. Cong. Pock Mech., Aachen, Balkema, 1991, p. 16-20

Fokker P.A., Kenter C.J., Rogaar H.P. - The effect of fluid pressure on the mechani- cal stability of rock salt. Proc. 7 th symp. on salt, Elsevier Sci. Pub., 1993.

Fries G. Berest P., Beaudoin B. - Eléments d'un inventaire des gisements de sel francais. Annales des mines, 1983, p. 39-46.

George B, Laporte P. - Exploitation de sel par dissolution à Hauterives (France). Rev. Industrje minérale-Mine, 1976, p. 219-226,

Ghoreychi M., Berest, Hardy H.R. Jr, Langer M. - The Mechanical Behavior of Salt. Proc. the Third Conference, Trans Tech Publisher, Germany, 1996.

Ghoreychi M. - Conséquences du comportement thermomécanique du sel pour la conception et la súreté d'un enfouissement de déchets radioactifs. Stockage en souterrain, Presses des Ponts et Chaussées. 1990, p. 229-243.

Ghoreychi M., Daupley X. - Inciciences du comportement mécanique et des processus de transport sur l'évolution à long terme des sites d'exploitation de sel, Journées nationales de géologie et de géotechnique (JNGG, 2002), Nancy, 2002.

Hunsche U. - Failure behaviour of rock salt around underground cavities. 7 th symp. on Rock Salt, Kakihana $\mathrm{H}$. (ed.), Elsevier B., Amsterdam, 1993.

Lai T.S. - Fluid flow through rock salt under various stress states, Ph.D. Civil Eng. Michigan State University, 1971.

Langer M. - The rheological behavior of rocksalt. Proc, First Conf. Mech. Behavior of Salt, Trans Tech Publications. Germany, 1981, p. 201-240.
Lemaitre J., Chaboche J.L. - Mécanique des matériaux solides. Dunod, Paris, 1988.

Marchal C. - Géométrie diu gîte évaporitique keuperien de la Lorraine-Champagne. Application à la reconnaissance de l'évolution paléogéographique du bassín de sédimentation. Sci. Terre. mém. 44, 1987.

Marchal P., Oger R., Plaid C., Romac M. Wéber A. - La mémoire du sel au pays đu Sânon. Les Foyers rurauxx « Au Pays du Sânon 1., 1994.

Meriaux M. Gannat E. - Connaissances actuelles sur la potasse en France. Annales des mines, 1980, p. 167-175.

Moretto R - Etude sédimentologique et gêochimique des dépôts de la série salffère paléngène du bassin de Bourg-enBresse (France). Sci. Teme, mém. 50, 1987.

Munson D.E., Dawson P.R. - A work hardening/recovery model of transient creep of salt during loading and unloading. Proc. 23rd US Symp. Rock Mech. 1982, p. 299-307.

Peach C.J.-Influence of deformation on the fluid transport properties of salt rocks. Ph.D. Univ. Utrecht, Geologica Ultraiectina, $n^{\circ} 77$. Hollande, 1991.

Spiers C.J., Peach C.J. - Development of dilatancy and permeability in rocks during creep: experiments on rock salt as rock analog. Int. Geol. Congr. Washington D.C. vol. 3, 1989, p. 162-173. 
Stokes R.J. - Mechanical properties of polycrystalline $\mathrm{NaCl}$. Int. Geol. Congr., Proc. British Ceramic Society, vol. 8-6, 1989, p. 189-207.

Stormont J.C., Howard C.L., Damen J.J.K. - Chances in rock salt peability due to nearby ecavation, Rock Mechanics as a multidisciplinary science. Ed. Roggiers, Rotterdam, 1991

Thorel L. - Plasticite et endommagement des roches ductiles - Application au sel gemme. Thèse de l'Ecole nationale des ponts et chaussees, 1994.
Thorel L... Ghoreychi M. - Plasticité et endommagement du sel gemme. Revue française de géotechnique, $\mathrm{n}^{\circ} 77,4^{\mathrm{c}}$ trimestre, 1996 .

Thorel L., Ghoreychi M. Cosenza P.. Chanchole S. - Damage and failure of Salt Rock under dry or wet conditions. 4th Conference on the Mechanica! Behavior of Salt, Montreal, Canada. 1996.

Vouille G., Tijani M., Grenier (de) F, - Expe. rimental determination of the rheological behavior of Tersanne rock salt. Proc. the First Conf on the Mechanical Behaviour of Salt. Pennsylvania State University, 1981.

Wallner M. - Stability calculation concerning a room and plillar design in rock salt. Proc. 5th Int. Cong. Rock Mech., Melburne, Australia, April 1983.

Wawersik W.R., Preece D.S. - Creep testing of salt, procedures, problems and suggestions. Proc. the First Conf. on the Mechanical Behaviour of Salt, Pennsylvania State University, 1981. 